\title{
Capacidade Discriminante do Inventário de Potencial de Abuso na Infância: Dados numa Amostra de Progenitores Portugueses
}

\author{
Discriminant Ability of the Child Abuse Potential Inventory: Data from a Sample \\ of Portuguese Parents
}

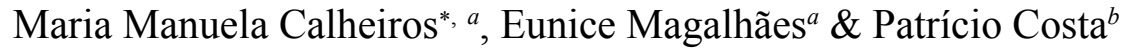 \\ ${ }^{a}$ Instituto Universitário de Lisboa (ISCTE-IUL), Cis-IUL, Lisboa, Portugal \\ \& ${ }^{b}$ ICVS/3B's Associated Laboratory, University of Minho, Braga, Portugal
}

\begin{abstract}
Resumo
Este estudo tem como objetivo testar a capacidade discriminante do Inventário de Potencial de Abuso na Infância (CAP), numa amostra de 641 progenitores portugueses. Trinta e sete por cento da amostra é constituída por progenitores maltratantes sinalizados às Comissões de Proteção de Crianças e Jovens e avaliados através do Questionário de Avaliação do Mau Trato, Negligência e Abuso Sexual. Os resultados indicaram que os progenitores maltratantes reportam valores significativamente superiores na Escala de Abuso e em todas as suas dimensões. Do ponto de vista da capacidade discriminante, a função da Escala de Abuso Global classificou corretamente 79,9\% e a função das subdimensões da Escala classificou corretamente $83,3 \%$ dos casos. Estes resultados revelam a potencialidade do instrumento na identificação de situações de potencial abuso na infância no contexto português.

Palavras-chave: Capacidade discriminante, Inventário Potencial Abuso, maus tratos.
\end{abstract}

\begin{abstract}
This study aims to test discriminant ability of the Child Abuse Potential Inventory (CAP) in a sample of 641 Portuguese parents. Thirty-seven percent of the sample is composed by abusive parents reported to the Comissions for the Protection of Children and Young People and evaluated by an assessment questionnaire of maltreatment, neglect and sexual abuse. Abusive parents showed significantly higher scores on the Abuse Scale and in all of its dimensions. Considering the discriminant analysis, the Abuse Scale correctly classified $79.9 \%$ of the cases and the function of sub dimensions correctly classified $83.3 \%$ of the cases. These results reveal the capacity of CAP in identifying cases of potential child abuse in the Portuguese context.

Keywords: Discriminant ability, Child Abuse Potential Inventory, maltreatment.
\end{abstract}

A avaliação de práticas parentais maltratantes tem sido alvo de crescente atenção empírica nas últimas décadas, no sentido de serem desenvolvidos esforços de intervenção e prevenção de situações de abuso futuro (e.g., Chaffin \& Valle, 2003; Milner, Robertson, \& Rogers, 1990). Neste contexto, o inventário Child Abuse Potential (CAP) tem sido largamente utilizado ao nível internacional decorrente das suas potencialidades na identificação de situações de potencial abuso físico na infância, perpetrado pelos cuidadores (Milner et al., 1990). O CAP, na sua versão original, envolve nove escalas: a Escala de Abuso (que se divide em 6 subescalas: 1 . Mau estar psicológico; 2. Rigidez, 3. Infelicidade, 4. Problemas com a criança e consigo mesmo; 5. Problemas com a família e 6. Problemas com outras pessoas), e três escalas de validade: Desejabilidade Social, Respostas aleatórias e Inconsistência. Têm sido de-

*Endereço para correspondência: Centro de Investigação e de Intervenção Social, Instituto Universitário de Lisboa, Av. das Forças Armadas, Lisboa, Portugal 1649-026. E-mail: maria.calheiros@iscte.pt senvolvidos diversos estudos de adaptação e validação do CAP em diferentes contextos, sendo que, genericamente, os resultados têm revelado bons índices de fidelidade e de validade de construto, convergente, preditiva e discriminante (e.g., Bringiotti, Barbich, \& De Paúl, 1998; De Paúl, Arruabarrena, \& Milner, 1993; Milner \& Crouch, 2012).

No que diz respeito à validade de constructo da Escala de Abuso, têm sido identificadas diferenças ao nível das dimensões obtidas em diferentes contextos, especificamente, no contexto Espanhol e Grego (i.e., 77 itens organizados em 5 dimensões) ou Chileno (i.e., 83 itens organizados em 7 dimensões; e.g., De Paúl et al., 1993; Diareme, Tsiantis, \& Tsitoura, 1997; Haz \& Ramirez, 2002). De salientar que as dimensões Mau Estar Psicológico e Rigidez são aquelas que tendem a manter-se mais estáveis entre os estudos (com exceção do estudo chileno), sendo que as dimensões relativas aos Problemas (com outras pessoas, com a família ou com a criança), e a Infelicidade são as que apresentam maior variabilidade entre as diferentes culturas.

Ao nível da fidelidade da escala, uma revisão recente de Milner e Crouch (2012) verificou que os valores obtidos em 
10 contextos culturais diferentes (i.e., Brasil, China, Espanha, Bélgica, Chile, Japão, Holanda, Croácia, Tailândia e Alemanha) variam entre 0,87 e 0,93 , o que sugere índices de consistência interna da Escala de Abuso elevados. Especificamente ao nível da língua portuguesa, um estudo com progenitores brasileiros (60 progenitores notificados no sistema de proteção e 60 não notificados) encontrou bons valores de consistência interna para a Escala Global e para as dimensões de Mau Estar Psicológico e Rigidez. No entanto, é de notar que os autores, ao replicarem a estrutura da escala obtida nos Estados Unidos da América, encontraram fragilidades ao nível da consistência interna das restantes dimensões: 0,32 (Infelicidade), 0,19 (Problemas com a criança e consigo mesmo), 0,48 (Problemas com a Família) e 0,61 (Problemas com os outros; Bérgamo, Pasian, Ávila de Mello, \& Bazon, 2009). Contudo, importa referir que os autores ao testarem a consistência interna das dimensões encontradas na versão original americana não consideraram potenciais especificidades do contexto ao nível da estrutura fatorial do inventário, em particular, na sua amostra de progenitores brasileiros.

Tendo em conta que este inventário avalia dimensões da parentalidade que refletem dificuldades de interação e problemas psicossociais dos progenitores, os estudos têm revelado a sua relação com variáveis como o abuso de substâncias (Ammerman, Kolko, Kirisci, Blackson, \& Dawes, 1999), dificuldades globais ao nível da saúde mental dos progenitores ou dificuldades dos indivíduos no reconhecimento das emoções das crianças (Asla, De Paúl, \& Pérez-Albéniz, 2011; Balge \& Milner, 2000). Estes resultados são congruentes com os modelos teóricos que sugerem que a presença de um conjunto de fatores individuais ou contextuais parece aumentar a probabilidade de o mau trato ocorrer (e.g., dificuldades socioeconómicas, história de abuso parental, ou competências parentais desadequadas; Belsky, 1993; Cicchetti \& Toth, 1995). Com efeito, de acordo com a teoria ecológica-desenvolvimental de Belsky (1993), a presença de múltiplos níveis de risco, desde os individuais aos socioambientais, deve ser analisada para melhor compreender os fatores que influem no mau trato infantil (Begle, Dumas, \& Hanson, 2010; Belsky, 1993). Além disso, Milner e Crouch (2012), na revisão anteriormente referida, verificaram que os fatores de risco encontrados nos estudos que utilizaram as versões adaptadas do inventário parecem ser congruentes com os obtidos na versão original, nomeadamente, a associação entre elevada pontuação na escala de abuso e história de abuso na infância, exposição a violência interparental, rejeição parental na infância, problemas de saúde mental (e.g., depressão e ansiedade), ou reduzidos níveis de satisfação com a vida.

Tendo como objetivo a identificação do potencial de abuso parental, os estudos desenvolvidos têm-se preocupado também com a capacidade de classificação e discriminação dos sujeitos com base nas pontuações obtidas na Escala de Abuso (Milner \& Crouch, 2012;
Robertson \& Milner, 1985). A revisão de Milner e Crouch (2012), anteriormente referida, revela que os estudos que reportam dados de análise discriminante revelaram, para a população geral, taxas de classificação corretas dos sujeitos que variam entre os $87 \%$ e os $100 \%$, em países como a Bélgica ou Holanda, sendo que os valores obtidos em amostras americanas variam entre $88 \%$ e $92 \%$. No caso dos progenitores maltratantes, a referida revisão revela que os valores obtidos com as versões traduzidas foram entre os $83 \%$ e os $100 \%$, sendo que nos EUA o valor foi de $92 \%$. Estes resultados vêm reforçar as potencialidades da Escala de Abuso enquanto instrumento com elevada capacidade diferenciadora das práticas parentais potencialmente abusivas.

Neste sentido, também no contexto português se afigura essencial avaliar as qualidades e potencialidades deste instrumento. Os dados preliminares de adaptação do CAP numa amostra de progenitores portugueses (Calheiros \& Magalhães, 2012) revelaram, ao nível da validade de construto, uma estrutura de cinco dimensões (1. Mau estar psicológico, 2. Rigidez, 3. Problemas com a criança e consigo mesmo, 4. Problemas com a Família e 5. Infelicidade e Problemas com outras pessoas), que é congruente com o modelo conceptual proposto por Milner e colaboradores (1990). No que se refere à fidelidade, os resultados revelaram valores de consistência interna adequados (Alfa de Cronbach): Escala de Abuso Global $(0,95)$, Mau Estar Psicológico $(0,94)$, Rigidez $(0,82)$, Problemas com a criança e consigo mesmo $(0,76)$, Problemas com a família $(0,81)$, e Infelicidade e Problemas com outras pessoas (0,76; Calheiros \& Magalhães, 2012). Quanto à validade convergente, os resultados obtidos no referido trabalho revelaram valores de correlação negativos e significativos entre as pontuações na Escala de Abuso e as obtidas na escala de Auto-Perceção para Adultos - Adult Self-Perception Profile - que avalia onze dimensões de auto-conceito (e.g., sociabilidade, competência no trabalho, moralidade, inteligência) e uma de auto-estima global (Messer \& Harter, 1986). Deste modo, considerando o potencial discriminante da escala de abuso do CAP largamente reportado ao nível das adaptações internacionais do instrumento e aqui descrito, e tendo em consideração as qualidades psicométricas da escala de abuso testada numa amostra de progenitores portugueses, iremos apresentar neste trabalho dados relativos à capacidade discriminante da Escala de Abuso, comparando um grupo de progenitores maltratantes e outro de progenitores não maltratantes.

\section{Método}

\section{Participantes}

O presente estudo incluiu 641 participantes $(75,4 \%$ do sexo feminino) com idades compreendidas entre os $17 \mathrm{e}$ os 76 anos $(M=38,12 ; D P=8,50)$. Em termos das habilitações literárias dos participantes, o nível do ensino superior apresentou a maior frequência na amostra $(31 \%)$, e pro- 
Calheiros, M. M., Magalhães, E. \& Costa, P. (2014). Capacidade Discriminante do Inventário de Potencial de Abuso na Infância: Dados numa Amostra de Progenitores Portugueses.

fissionalmente, as profissões técnicas e científicas surgem em maior percentagem $(24,8 \%)$, sendo os trabalhadores não qualificados a menor percentagem $(13,4 \%)$. Os dados foram recolhidos em diferentes zonas de Portugal e diferentes instituições, nomeadamente, em comissões de proteção de crianças e jovens, em escolas públicas e instituições comunitárias. Foram definidos dois grupos de progenitores no presente estudo: "Progenitores Maltratantes" versus "Progenitores Não Maltratantes". No sentido de analisar a capacidade discriminante do CAP, foi inicialmente considerada a variável "Sinalização" nos serviços de promoção e proteção (Sinalizados: $N=263$; Não sinalizados: $N=$ 395) para a diferenciação dos progenitores em dois grupos. Não obstante, dado que a sinalização dos progenitores nos serviços de proteção pode ser consequência de diversas situações de perigo/risco, esta variável poderia não ser a mais adequada para a análise discriminante dos itens. Por esse motivo, recorremos ao resultado no Questionário de Avaliação do Mau Trato, Negligência e Abuso Sexual
(Calheiros, 2006) para definir o grupo de "Progenitores Maltratantes", sendo que apenas os progenitores que pontuavam na dimensão "Mau Trato" deste questionário foram incluídos neste grupo. Desta forma foram retidos 246 dos progenitores sinalizados, o que corresponde a $38,4 \%$ do total da amostra. Dezassete progenitores sinalizados, mas que não preenchiam o critério em termos de pontuação na dimensão de Mau Trato no referido questionário foram eliminados da análise. Os "Progenitores Não Maltratantes" foram selecionados por conveniência em contexto comunitário sem histórico de registo no sistema de proteção. No que diz respeito às características sociodemográficas dos dois grupos de progenitores (Tabela 1), verificamos que os grupos se diferenciam significativamente em todas as dimensões aqui analisadas. Assim, verificamos que o grupo de progenitores maltratantes é constituído por mais participantes do sexo feminino, bem como por progenitores mais novos, constituição familiar sobretudo monoparental, e em situação de desemprego, quando comparado com o grupo de progenitores não maltratantes.

Tabela 1

Características Sociodemográficas dos Participantes em Função do Grupo (N=641)

\begin{tabular}{lcccc}
\hline Variáveis & & $\begin{array}{c}\text { Progenitores Não Maltratantes } \\
(n=395)\end{array}$ & $\begin{array}{c}\text { Progenitores Maltratantes } \\
(n=246)\end{array}$ & $t_{(614)} / \chi^{2}(1)$ \\
\hline Idade & & $M=38,98 ; D P=7,47$ & $M=36,64 ; D P=9,85$ & $3,29^{*}$ \\
& & Frequência (\%) & Frequência (\%) & \\
Sexo & Feminino & $286(73,9)$ & $197(88,7)$ & $18,93^{* *}$ \\
& Masculino & $101(26,1)$ & $25(11,3)$ & \\
Tipo de Família & Monoparental & $51(13)$ & $131(61,2)$ & $153,57^{* *}$ \\
& Biparental & $342(87)$ & $83(38,8)$ & $105,92^{* *}$ \\
Situação & Ativo & $329(86,6)$ & $84(45,7)$ & \\
\hline
\end{tabular}

${ }^{*} p<0,01 ; * * p<0,001$.

\section{Instrumentos}

Inventário de Potencial de Abuso. No presente estudo foi utilizada uma versão do instrumento original traduzida para português. Este inventário, destinado a progenitores, é constituído por 160 itens de resposta dicotómica ("Concordo" ou "Não Concordo"), estando organizados em nove escalas, na sua versão original: a Escala de Abuso (que se divide nas 6 subescalas anteriormente referidas: 1. Mau estar psicológico; 2. Rigidez, 3. Infelicidade, 4. Problemas com a criança e consigo mesmo; 5 . Problemas com a família e 6. Problemas com outras pessoas), e três escalas de validade: Desejabilidade Social, Respostas aleatórias e Inconsistência, que se destinam à validação dos resultados obtidos, detetando se os progenitores responderam no sentido do socialmente esperado, se responderam aleatoriamente, ou se foram inconsistentes nas respostas dadas. A combinação das pontuações destas três escalas resulta em três Índices de Validade: Imagem Positiva, Imagem Negativa e Resposta Aleatória (Milner \& Crouch, 2012). A pontuação total de cada escala é obtida através da soma das pontuações correspondentes aos itens que a compõem. A administração foi realizada de acordo com os procedimentos sugeridos pelos autores da versão original, que referem que a utilização correta do Inventário requer que este seja aplicado no formato que inclui os 160 itens, sendo que qualquer versão reduzida do Inventário não pode ser considerada válida (Milner et al., 1990).

Questionário de Avaliação do Mau Trato, Negligência e Abuso Sexual (Calheiros, 2006). Este instrumento destina-se a técnicos e tem como objetivo a avaliação de diferentes tipos de mau trato e negligência em crianças e adolescentes. Avalia 21 itens organizados em 5 dimensões: 
mau trato (interação física agressiva, métodos de violência física, interação verbal agressiva, métodos de disciplina coercivos/punitivos, padrões de avaliação hostis e negativistas), negligência física (vestuário, higiene e bem estar físico, condições de habitabilidade e higiene, alimentação, acompanhamento na saúde física), negligência educacional (necessidades de desenvolvimento, acompanhamento da saúde mental da criança, acompanhamento escolar), falta de supervisão (acompanhamento alternativo suplementar, segurança no meio, supervisão, desenvolvimento sociomoral, relação com as figuras de vinculação) e abuso sexual. Esta medida apresentou, no seu estudo original, boa consistência interna, revelando os seguintes valores para o coeficiente de Alfa de Cronbach: mau trato $(0,86)$; negligência física $(0,86)$, negligência educacional $(0,76)$, e falta de supervisão $(0,73)$.

\section{Procedimentos}

Os questionários foram recolhidos em comissões de proteção de crianças e jovens (CPCJ) em situação de risco/perigo em Portugal, bem como em escolas públicas e instituições comunitárias, entre 2009 e 2011. Os dados foram recolhidos no âmbito de um projeto de investigação mais alargado a decorrer no Centro de Investigação e Intervenção Social (CIS-ISCTE-IUL). Do ponto de vista ético, foi solicitada uma autorização formal às instituições para a recolha de dados junto dos sujeitos, tendo sido posteriormente recolhido o consentimento informado junto dos progenitores para a participação no presente estudo.

\section{Análise de Dados}

Foram realizadas inicialmente análises descritivas da Escala de Abuso e das suas dimensões ao nível da sensibilidade (i.e., medidas de forma). No sentido de identificar o potencial discriminante da Escala de Abuso entre os progenitores maltratantes e não maltratantes foi realizada uma Análise Discriminante para o valor obtido na Escala de Abuso Global, e para as suas subdimensões. De facto, a análise discriminante é usada para identificar de que forma determinadas características contribuem para a discriminação entre dois ou mais grupos, bem como determinar a forma mais parcimoniosa para os distinguir (Burns \& Burns, 2009). Do mesmo modo, a utilização desta análise permite classificar sujeitos em grupos, providenciando dados relativamente à capacidade da função de distinguir os grupos (ao contrário da estatística qui quadrado, que apenas nos fornece significância estatística; Burns \& Burns, 2009). De acordo com os estudos realizados neste domínio, a Análise Discriminante parece ser a mais adequada na classificação de sujeitos, quando comparada com outro tipo de análises de dados (Pohar, Blas, \& Turk, 2004), sendo particularmente eficiente quando se trata de grupos de dimensões muito diferentes (como é o caso do presente estudo, onde os progenitores não maltratantes são 395 e os progenitores maltratantes são 246; Maroco, 2010). A significância a considerar na análise discriminante deverá ter em conta o número de variáveis que estamos a comparar $(\alpha / p$, sendo $p$ o número de variáveis -5 no caso das subdimensões $-\operatorname{logo} 0,05 / 5=0,01$ será o valor alfa a considerar na decisão).

\section{Resultados}

\section{Análises Descritivas}

No que se refere às medidas de forma - Assimetria e Achatamento - da Escala de Abuso Global e das suas subdimensões, os dados obtidos revelaram valores absolutos inferiores a 3 para a Assimetria e 7 para o Achatamento, refletindo o que Kline (2005) sugere como valores não problemáticos que permitem testar modelos lineares generalizados. Tal como podemos observar na Tabela 2, a assimetria das distribuições de todas as dimensões indica que todas apresentaram um enviesamento positivo (ou uma assimetria à direita). A análise do achatamento revelou que a dimensão "Problemas com a criança e consigo mesmo" assume uma distribuição leptocúrtica (i.e., valor $>0$ ), sendo que todas as outras dimensões assumem uma distribuição platicúrtica (i.e., valor $<0$ ).

Tabela 2

Estatística Descritiva da Escala de Abuso e Subescalas, Média e Desvio-Padrão (N=641)

\begin{tabular}{lcccc}
\hline & Média & Desvio Padrão & Assimetria & Achatamento \\
\hline Escala de Abuso Global & 28,73 & 16,54 & 0,54 & $-0,71$ \\
Mau Estar Psicológico & 7,46 & 6,99 & 0,72 & $-0,74$ \\
Problemas com a família & 3,31 & 3,03 & 0,81 & $-0,37$ \\
Infelicidade e problemas com outras pessoas & 6,33 & 3,31 & 0,26 & $-0,60$ \\
Problemas com a criança e consigo mesmo & 2,76 & 2,43 & 1,35 & 1,56 \\
Rigidez & 8,86 & 4,32 & 0,19 & $-0,83$ \\
\hline
\end{tabular}


Calheiros, M. M., Magalhães, E. \& Costa, P. (2014). Capacidade Discriminante do Inventário de Potencial de Abuso na Infância: Dados numa Amostra de Progenitores Portugueses.

Diferenças entre Progenitores Maltratantes e Não Maltratantes ao Nivel das Pontuações no CAP

No que diz respeito à pontuação obtida pelos progenitores maltratantes e não maltratantes na Escala de Abuso Global e nas suas subdimensões, verificámos que os grupos se diferenciam significativamente em todas as dimensões (Tabela 3). Os progenitores maltratantes apresentam pontuações significativamente mais elevadas em todas as dimensões, com tamanhos de efeito muito elevados $(>1)$ em todas as dimensões e elevado $(0,89)$ na dimensão Infelicidade e Problemas com outras pessoas (Maroco, 2010).

Tabela 3

Diferenças de Médias entre Progenitores Maltratantes e Não Maltratantes

\begin{tabular}{lcccc}
\hline Escala de Abuso & $\begin{array}{c}\text { Progenitores } \\
\text { Não Maltratantes }(n=395) \\
M(D P)\end{array}$ & $\begin{array}{c}\text { Progenitores } \\
\text { Maltratantes }(n=246) \\
M(D P)\end{array}$ & $t(639)$ & $d$ de Cohen \\
\hline Escala de Abuso Global & $20,60(11,56)$ & $41,80(14,87)$ & $-20,19^{*}$ & 1,56 \\
Mau Estar Psicológico & $4,70(5,52)$ & $12,41(7,18)$ & $-14,60^{*}$ & 1,20 \\
Problemas com a família & $2,04(2,16)$ & $5,35(3,10)$ & $-15,94^{*}$ & 1,24 \\
Infelicidade e Problemas com outras pessoas & $5,16(2,82)$ & $7,70(2,89)$ & $-12,80^{*}$ & 0,89 \\
Problemas com a criança e consigo mesmo & $1,59(1,29)$ & $4,65(2,63)$ & $-19,63^{*}$ & 1,48 \\
Rigidez & $7,10(3,67)$ & $11,69(3,75)$ & $-15,26^{*}$ & 1,24 \\
\hline
\end{tabular}

${ }^{*} p<0,001$.

\section{Capacidade Discriminante da Escala de Abuso}

Dado que a Escala de Abuso Global é constituída pelo somatório de todos os itens das suas subdimensões, foram realizadas duas análises discriminantes: uma para a pontuação global e outra para as 5 dimensões. A análise da Escala de Abuso Global extraiu uma função discriminante retendo a pontuação da Escala de Abuso Global como estatisticamente significativa, e o mesmo foi observado para a função discriminante com as 5 dimensões (Tabela 4).

Tabela 4

Resultados da Função Discriminante com a Escala de Abuso Global e com as Cinco Dimensões (N=641)

\begin{tabular}{lcccc}
\hline Função Discriminante & Eigenvalue & $\begin{array}{c}\text { Correlação } \\
\text { Canónica }\end{array}$ & $\begin{array}{c}\text { Lambda } \\
\text { de Wilk }\end{array}$ & $p$ \\
\hline Função com a Escala de Abuso Global & 0,638 & 0,624 & 0,610 & $<0,001$ \\
Função com as cinco dimensões da Escala de Abuso & 0,839 & 0,675 & 0,544 & $<0,001$ \\
\hline
\end{tabular}

Com efeito, podemos observar na Tabela 4 que o grupo de progenitores maltratantes se diferencia significativamente do grupo de progenitores não maltratantes pela função da Escala de Abuso Global [Wilks's $\Lambda=0,610$; $\left.\chi^{2}(1)=315,092, p<0,001\right]$, bem como pela função com as cinco dimensões [Wilks's $\Lambda=0,544 ; \chi^{2}(5)=387,702, p<$ $0,001]$. A correlação canónica apresentada reflete uma correlação múltipla entre a Escala de Abuso Global utilizada na análise e a função discriminante de 0,624 , sendo este modelo capaz de explicar $39 \%$ da variação entre os grupos. A correlação canónica das subdimensões e a função discriminante foi de 0,675 , sendo este modelo capaz de explicar $46 \%$ da variação entre os grupos. No que diz respeito à classificação dos indivíduos, no caso da Escala de Abuso
Global, 79,9\% dos casos foram corretamente classificados [um acréscimo de $26,93 \%$ comparado com a percentagem de classificação pelo acaso: $(246 / 641)^{2}+(395 / 641)^{2} \times 100=$ $52,97 \%]$. Setenta e três por cento dos progenitores maltratantes foram classificados corretamente (Sensibilidade), e $84,1 \%$ dos não maltratantes (Especificidade). No caso da função discriminante com base nas subdimensões, $83,3 \%$ dos casos foram corretamente classificados [um acréscimo de $30,23 \%$ comparado com a percentagem de classificação pelo acaso: $(246 / 641)^{2}+(395 / 641)^{2}$ x 100 $=52,97 \%$ ] O modelo das subdimensões classificou corretamente $74,8 \%$ dos progenitores maltratantes (Sensibilidade), e $88,4 \%$ dos não maltratantes (Especificidade). Dado que pretendemos um modelo discriminante que tenha validade 
externa e interna, foi utilizada a técnica de "leave-one-out classification" como forma de validação cruzada (Tabela 5). Conforme podemos constatar, a precisão de classificação relativa à análise discriminante é similar à precisão de classificação proveniente da validação cruzada, o que remete para a qualidade, estabilidade e generalização das funções discriminantes utilizadas.

Tabela 5

Resultados da Classificação pelo Método de CrossValidation

\begin{tabular}{ccc}
\hline & \multicolumn{2}{c}{ Grupo Predito } \\
\cline { 2 - 3 }$N=641(100 \%)$ & $\begin{array}{c}\text { Não Maltratantes } \\
\end{array}$ & Maltratantes \\
& $n(\%)$ & $n(\%)$ \\
\hline
\end{tabular}

Modelo Global

Não Maltratantes

$n=395(100 \%)$

$332(84,1)$

$63(15,9)$

Maltratantes

$n=246$ (100\%)

$66(26,8)$

$180(73,2)$

Modelo Subdimensões

Não Maltratantes

$n=395$ (100\%)

$349(88,4)$

$46(11,6)$

Maltratantes

$n=246(100 \%)$

$62(25,2)$

$184(74,8)$

\section{Discussão}

Na sequência de estudos prévios centrados nas características psicométricas da Escala de Abuso numa amostra portuguesa (Calheiros \& Magalhães, 2012), o presente trabalho pretendia explorar as suas potencialidades no que diz respeito à capacidade discriminativa de progenitores maltratantes no contexto português. Foram inicialmente realizadas análises de comparação entre o grupo de progenitores maltratantes e o grupo de não maltratantes em dimensões sociodemográficas que têm sido reportadas na literatura enquanto fatores de risco para as práticas maltratantes (e.g., condições socioeconómicas; e.g., Connelly $\&$ Straus, 1992). Os resultados revelaram que o grupo de progenitores maltratantes é composto por progenitores mais novos, com uma percentagem mais elevada de desemprego e de uma estrutura familiar monoparental, comparativamente aos não maltratantes. Com efeito, o que a literatura sugere é que o risco de mau trato parece aumentar na presença de um inadequado suporte por parte da rede social (o que pode acontecer num contexto de monoparentalidade; Connelly \& Straus, 1992), de reduzidas condições socioeconómicas do agregado familiar (podendo o desemprego ser aqui perspetivado enquanto condição potenciadora de dificuldades socioeconómicas; Berger, 2005), ou ainda quando há um conhecimento e experiência insuficientes no que diz respeito aos cuidados a prestar às crianças (o que pode acontecer em progenitores mais novos; Connelly \& Straus, 1992). No entanto, importa salientar que, no que diz respeito às diferenças obtidas ao nível da idade, a média em ambos os grupos situa-se na mesma faixa etária (isto é nos 30 anos), sendo a diferença entre as médias de aproximadamente 2 anos. Ao passo que, os estudos que têm refletido acerca das implicações sérias da idade enquanto fator de risco para o mau trato têm-se centrado essencialmente nas dificuldades associadas à parentalidade na adolescência, que não é de todo a realidade aqui analisada e discutida (e.g., Dukewich, Borkowski, \& Whitman, 1996). No que diz respeito ao sexo dos participantes, o grupo de progenitores maltratantes apresenta uma maior percentagem de participantes do sexo feminino $(88,7 \%)$ comparativamente ao grupo de progenitores não maltratantes $(74,4 \%)$. Sidebotham e Golding (2001) estudaram esta dimensão sociodemográfica, e verificaram que os fatores de risco das mães se diferenciam dos apresentados pelos pais, sendo que as mães parecem apresentar um número superior e diferenciado de fatores de risco (i.e., idade inferior aos 20 anos, reduzidas habilitações educacionais, história de abuso sexual, história psiquiátrica e ausência de uma figura parental na infância) comparativamente aos pais (idade inferior aos 20 anos, reduzidas habilitações educacionais, história psiquiátrica e ter sido cuidado pelos serviços de proteção na infância; Sidebotham \& Golding, 2001).

Posteriormente foram analisadas as diferenças de médias na escala de abuso e nas suas dimensões entre progenitores maltratantes e não maltratantes. Os resultados revelam que os progenitores maltratantes tendem a reportar valores significativamente superiores na Escala de Abuso e em todas as suas dimensões. Dado que foram encontradas diferenças estatisticamente significativas do ponto de vista da análise de comparação de médias, procedemos à Análise Discriminante no sentido de verificar se a escala de abuso e as suas dimensões apresentam um elevado potencial discriminante de progenitores maltratantes e de não maltratantes. Os resultados obtidos suportam a capacidade da Escala de Abuso para discriminar estes dois grupos no contexto duma amostra de progenitores portugueses, tal como sustentado pela função discriminante que reteve a pontuação da Escala de Abuso Global e suas dimensões como estatisticamente significativas. Com efeito, pudemos observar que a função da Escala de Abuso Global classificou corretamente $79,9 \%$ dos casos e que a função das subdimensões classificou corretamente $83,3 \%$ dos casos. Estes resultados, embora ligeiramente inferiores, aproximam-se dos dados obtidos nos estudos revistos por Milner e Crouch (2012) que reportam valores entre os $87 \%$ e os $100 \%$. Podemos verificar que a precisão da classificação utilizando as subdimensões é maior quando comparada com a que tem em conta apenas a pontuação global. Em ambas as funções, garantimos que a proporção de classificações corretas foi superior em mais de $25 \%$ à 
Calheiros, M. M., Magalhães, E. \& Costa, P. (2014). Capacidade Discriminante do Inventário de Potencial de Abuso na Infância: Dados numa Amostra de Progenitores Portugueses.

percentagem proporcional de classificações corretas devido ao acaso. A análise dos falsos negativos revela-se particularmente pertinente no presente trabalho, uma vez que este inventário tem como objetivo avaliar o potencial de abuso dos progenitores. Com efeito, recorrendo ao modelo com a Escala Global ficam por identificar $26,8 \%$ dos progenitores maltratantes, sendo que considerando as subdimensões, ficam $25,2 \%$ por identificar. Este resultado sugere assim a necessidade de triangular métodos de avaliação das práticas parentais (e.g., observação, entrevistas, consulta de outros profissionais que acompanham as famílias) com vista à identificação correta de progenitores potencialmente maltratantes.

A comparação dos resultados encontrados com os dois modelos sugere que a utilização das subdimensões para discriminar os grupos permite obter uma melhor classificação dos casos e uma menor percentagem de casos negativos. Do ponto de vista da utilização deste instrumento em contexto de avaliação, estes resultados sugerem que a análise das subdimensões pode fornecer informação detalhada e importante ao profissional, nomeadamente, acerca das áreas mais problemáticas nestes progenitores (e.g., problemas relacionais com elementos familiares ou com a criança, padrão de pensamento rígido) e que fornecem pistas importantes para a intervenção e prevenção.

Não obstante a relevância dos resultados aqui discutidos, importa notar limitações associadas ao presente estudo, designadamente, ao nível do desequilíbrio em termos do número de progenitores que constituem os grupos de maltratantes e de não maltratantes. No futuro seria interessante testar estes resultados com uma amostra mais equilibrada deste ponto de vista. Não obstante, os resultados aqui apresentados e discutidos revelam-se teoricamente plausíveis, uma vez que reforçam também as qualidades psicométricas deste instrumento. Assim, estes dados no contexto de uma amostra de progenitores portugueses contribuem para a discussão internacional acerca das potencialidades do CAP enquanto instrumento que permitirá a identificação de situações de potencial de abuso pelos sistemas de proteção das crianças e jovens.

\section{Referências}

Asla, N., De Paúl, J., \& Pérez-Albéniz, A. (2011). Emotion recognition in fathers and mothers at high-risk for child physical abuse. Child Abuse \& Neglect, 35(9), 712-721. doi:10.1016/j. chiabu.2011.05.010

Ammerman, R. T., Kolko, D. J., Kirisci, L., Blackson, T. C., \& Dawes, M. A. (1999). Child abuse potential in parents with histories of substance use disorder. Child Abuse \& Neglect, 23(12), 1225-1238. doi:10.1016/S0145-2134(99)00089-7

Balge, K. A., \& Milner, J. S. (2000). Emotion recognition ability in mothers at high and low risk for child physical abuse. Child Abuse and Neglect, 24, 1289-1298. doi:10.1016/S01452134(00)00188-5
Begle, A., Dumas, R., \& Hanson, J. (2010). Predicting child abuse potential: An empirical investigation of two theoretical frameworks. Journal of Clinical Child \& Adolescent Psychology, 39(2), 208-219. doi:10.1080/15374410903532650

Belsky, J. (1993). Etiology of child maltreatment: A developmental-ecological analysis. Psychological Bulletin, 114, 413-434.

Bérgamo, L., Pasian, S., Ávila de Mello, I., \& Bazon, M. (2009). O Inventário de Potencial de Maus-Tratos Infantil: Estudo de precisão e validade. Avaliação Psicológica, 8(3), 425-435.

Berger, L. (2005). Income, family characteristics, and physical violence toward children. Child Abuse \& Neglect, 29, 107133. doi:10.1016/j.chiabu.2004.02.006

Bringiotti, M., Barbich, A., \& De Paúl, J. (1998). Validacion de una version preliminar del Child Abuse Inventory para su uso en Argentina. Child Abuse \& Neglect, 22(9), 881-888. doi:1016/S0145-2134(98)00067-2

Burns, R. P., \& Burns, R. A. (2009). Discriminant analysis. In R. P. Burns \& R. A. Burns (Eds.), Business research methods and statistics using SPSS. London: Sage.

Calheiros, M. (2006). A construção social do mau trato e negligência parental: Do senso comum ao conhecimento científico. Lisboa, Portugal: Fundação Calouste Gulbenkian.

Calheiros, M. M., \& Magalhães, E. (2012). Inventário de Potencial de Abuso na Infância: Dados preliminares das suas qualidades psicométricas numa amostra portuguesa. Revista Laboratório de Psicologia,10(1),63-79.

Chaffin, M., \& Valle, L. A. (2003). Dynamic prediction characteristics of the Child Abuse Potential Inventory. Child Abuse \& Neglect, 27, 463-481. doi:10.1016/S0145-2134(03)00036-X

Cicchetti, D., \& Toth, S. L. (1995). A developmental psychopathology perspetive on child abuse and neglect. Journal of the American Academy of Child and Adolescent Psychiatry, 34, 541-565. doi:10.1097/00004583-199505000-00008

Connelly, C., \& Straus, M. (1992). Mother's age and risk for physical abuse. Child Abuse \& Neglect, 16, 709-718. doi:10.1016/0145-2134(92)90107-3

De Paúl, J., Arruabarrena, M. I., \& Milner, J. (1993). Manual de utilizacion e interpretacion. Inventario de Potencial de Maltrato Infantil. San Sebastián, España: Libreria Zorroaga.

Diareme, S., Tsiantis, J., \& Tsitoura, S. (1997). Cross-cultura validation of the Child Abuse Potential Inventory in Greece: A preliminary study. Child Abuse \& Neglect, 21(11), 10671079. doi:10.1016/S0145-2134(97)00066-5

Dukewich, T. L., Borkowski, J. G., \& Whitman, T. L. (1996). Adolescent mothers and child abuse potential: An evaluation of risk factors. Child Abuse \& Neglect, 20(11), 1031-1047. doi:10.1016/0145-2134(96)00093-2

Haz, A., \& Ramirez, R. (2002). Adaptacion del Child Abuse Potential Inventory en Chile: analisis de las dificultades y desafios de su aplicacion a partir de dos estudios Chilenos. Child Abuse \& Neglect, 26, 481-495. doi:10.1016/S01452134(02)00324-1

Kline, R. (2005). Principles and practice of structural equations modeling ( $2^{\text {nd }}$ ed. $)$. London: Guilford.

Maroco, J. (2010). Análise de equações estruturais. Fundamentos teóricos, software e aplicações. Pero Pinheiro, Portugal: Report Number.

Messer, B., \& Harter, S. (1986). Manual for the adult self perception profile. Unpublished manuscript, University of Denver, CO.

Milner, J. S., \& Crouch, J. (2012). Psychometric characteristics of translated versions of the Child Abuse Potential Inventory. Psychology of Violence, 2(3), 239-259. doi:10.1037/a0026957 
Milner, J. S., Robertson, K. R., \& Rogers, D. L. (1990). Childhood history of abuse and adult child abuse potential. Journal of Family Violence, 5(1), 15-34. doi:10.1007/BF00979136

Pohar, M., Blas, M., \& Turk, S. (2004). Comparison of logistic regression and linear discriminant analysis: A simulation study. Metodološki Zvezki, 1(1), 143-161. Retrieved from http://mrvar.fdv.uni-lj.si/pub/mz/mz1.1/pohar.pdf

Robertson, K., \& Milner, J. (1985). Convergent and discriminant validity of the Child Abuse Potential Inventory. Journal of Personality Assessment, 49(1), 86-88. doi:10.1207/ s15327752jpa4901_16

Sidebotham, P., \& Golding, J. (2001). Child maltreatment in the "children of the nineties": A longitudinal study of parental risk factors. Child Abuse \& Neglect, 25(9), 1177-1200. doi:10.1016/S0145-2134(01)00261-7 